\title{
Abdominal Aortic Aneurysms in Acute Coronary Syndrome: a
}

\section{Comparison}

\section{Ebrahim Haidary Sardaby ${ }^{1}$, Mohammad Ghasem Hanafi ${ }^{2}$, Nehzat Akiash ${ }^{1}$, Anahid Makvandi Alimoradi ${ }^{3 *}$}

'Department of Cardiology, School of Medicine, Atherosclerosis Research Center, Razi Hospital, Ahvaz Jundishapur University of Medical Sciences, Ahvaz, Iran

${ }^{2}$ Department of Radiology, School of Medicine, Imam Khomeini Hospital, Golestan Hospital, Ahvaz Jundishapur University of Medical Sciences, Ahvaz, Iran

${ }^{3}$ Department of Cardiology, School of Medicine, Imam Khomeini Hospital, Ahvaz Jundishapur University of Medical Sciences, Ahvaz, Iran.

Study Area:Ahvaz, Iran

Coordinates:31 $19^{\prime} 13^{\prime \prime N}$; 48 $40^{\circ}$ o9"E

Keywords: Echocardiography, Ultrasonography, Diagnostic Imaging.

\section{Introduction:}

An abdominal aortic aneurysm is one of the diseases which need high cost to cure in advanced countries. It generally occurs in men of above $65 \mathrm{yrs}$. According to an assessment in the United States, the disease is the 13th cause of mortality in this country (Sampson et al., 2014). Although some patients with this symptom experience vague and nonspecific symptoms such as back and abdominal pain, but most of the patients are asymptomatic, as long as these aneurysms are ruptured, which can lead to death in $65-85 \%$ of cases (Bush et al., 2003).

Although aortic aneurysms may occur in any part of the sub-diaphragmatic aorta, due to its high prevalence in some areas, many researchers use abdominal aortic aneurysm for aorta under the renal artery (Buck et al., 2016). The normal diameter of the aorta differs in different regions depending on the age, sex and body weight, and it reduces as the artery progresses in the abdominal cavity, bifurcates as the iliac arteries. The diameter of the infrarenal aorta in older men is between 15-24 $\mathrm{mm}$ (Kontopodis et al., 2016). In a study by McGregor etal., the diameter of the abdominal aorta greater than $30 \mathrm{~mm}$ has been considered as an aortic aneurysm.

\section{Abstract}

This is a cross-sectional study aims to evaluate and compare the accuracy rate between ultrasonography and transthoracic echocardiography. Patients with ACS and had inclusion criteria were included. Patients underwent abdominal ultrasonography by an experienced radiologist, and the condition of the abdominal aorta was examined. The patients underwent transthoracic echocardiography by an experienced attending cardiologist, and the results of the abdominal aorta were evaluated and recorded. We found a significant relationship between hypertension of patients and abdominal aortic aneurysm prevalence. There is no significant relationship among the type of ACS, smoking, left ventricle size and the prevalence of abdominal aortic aneurysm. The results of ultrasound and echocardiography showed that all three patients have abdominal aortic aneurysms in two groups were diagnosed similarly with abdominal aortic aneurysms, so the accuracy of diagnosis, sensitivity, and specificity of patients were $100 \%$. Conclusively, the transthoracic echocardiography, a diagnostic screening test, can be a suitable substitute for ultrasonography in the diagnosis of abdominal aortic aneurysm in patients with the acute coronary syndrome.

According to the US Association and Committee of Cardiovascular Surgeons, the aneurysm will occur if the diameter of the abdominal aorta is 1.5 times greater than normal (McGregor et al., 1975).

Currently, the most common and important method for screening this disease is abdominal ultrasonography. There are several different guidelines to assess the age and conditions for doing this (Kostun \& Malik, 2016). Some of these guidelines suggest that every man; aged between 65 and 75 years old should refer for screening at least once. Men and women (younger than 65 years) without a history of smoking did not need screening, but if they had a positive smoking history in the family, they would be recommended taking part in the program even before the age of 65 (Hultgren, 2013).

Echocardiography is one of the other diagnostic methods for aortic aneurysms, and it has been well documented in the diagnosis of this disease in various studies. Transthoracic echocardiography was done for all patients with ACS, so the current study aims to evaluate the accuracy rate of two methods of abdominal ultrasonography and transthoracic echocardiography and compare them with each other. 


\section{Materials and methods:}

This cross-sectional study was done to compare two methods of ultrasonography (as a method used in current guidelines for screening abdominal aortic aneurysms) and transthoracic echocardiography in the diagnosis of abdominal aortic aneurysm. The subjects were selected from patients with ACS admitted to Imam Khomeini Hospital in Ahvaz. We included the patients between 18 and 80 years old with ACS received informed consent to enter the study. A history with previous abdominal aneurysm, repaired abdominal aortic aneurysm with any previous procedure given no consent were excluded from the study.

First, a form including demographic information, history of previous illnesses and family history of various diseases were filled up from the subjects. An experienced attending radiologist examined and recorded the status of abdominal aorta of patients undergoing an abdominal ultrasound. The patient underwent transthoracic echocardiography by attending cardiologist, and the results of abdominal aorta were evaluated and recorded. For the accuracy rate of procedures to be constant with the standard quality of the US, echocardiography, and ultrasonography performed during the study were conducted by a professor of radiological division on the above groups. Then, the information on the first form and the results of the echocardiographic findings were collected.

Results were reported using descriptive statistics including mean, standard deviation, frequency, and frequency percentage. Also, for the correlation between the true parameters of the study and its prevalence, Pearson correlation coefficient test was used in the case of normal data, and Spearman correlation coefficient was used in the case of abnormal data. The specif icity was reported to check the diagnostic accuracy with true positive, true negative, false positive and false negative. The analysis was performed using SPSS, and the significance level was considered to be less than 0.05 .

\section{Results:}

The mean age of 22 patients was $65.84 \pm 5.75$ years $(45-77)$. The left ventricle size of patients was $4.94 \pm 0.39 \mathrm{~cm}(4.2-$ 5.9\%) (Table-1).

Patients' aortic size was $21.27 \pm 3.59 \mathrm{~mm}(17-38)$ based on ultrasonography, and $21.35 \pm 3.39 \mathrm{~mm}$ (16-25) based on echocardiography. There was a significant direct relationship between aortic sizes in ultrasonography and transthoracic echocardiography in patients with the acute coronary syndrome $(\mathrm{p}<0.001)$ (Table-2) (Fig.- 1). There was a significant relationship between hypertension of patients and abdominal aortic aneurysm $(\mathrm{p}<0.042)$. There was no significant relationship among the type of ACS, smoking, left ventricle size and the prevalence of abdominal aortic aneurysms (Table-3). The results of ultrasound and echocardiography showed that all three patients have abdominal aortic aneurysms were diagnosed commonly in two groups of abdominal aortic aneurysms, so the accuracy of diagnosis, sensitivity, and specificity of patients were $100 \%$.

Table 1. Patient's characteristics

\begin{tabular}{|c|c|c|}
\hline Group Variable & Patients $(\mathrm{n}=122)$ & \\
\hline Age, years, Mean \pm SD $(R$ & nge) & $65.84 \pm 5.75(45-77)$ \\
\hline Sex, $\mathrm{n}(\%)$ & Male & $51(41.8)$ \\
\hline & Female & $71(58.2)$ \\
\hline Type of ACS, n (\%) & UA & $82(67.2)$ \\
\hline & NSTEMI & $22(18)$ \\
\hline & ExtensiveSTEMI & $8(6.6)$ \\
\hline & Anterior STEMI & $6(4.9)$ \\
\hline & InferiorSTEMI & $4(3 \cdot 3)$ \\
\hline Hypertension & Yes, n (\%) & $43(35.2)$ \\
\hline & No, n (\%) & $79(64.8)$ \\
\hline Smoking & Yes, n (\%) & $35(28.7)$ \\
\hline & No, n (\%) & $87(71.3)$ \\
\hline Left ventricle diameter & $4 \cdot 5=$ & $28(23)$ \\
\hline$(\mathrm{cm})$ & $4 \cdot 5-5$ & $47(38.5)$ \\
\hline & $5-5 \cdot 5$ & $43(35.2)$ \\
\hline & $>5$ & $4(3 \cdot 3)$ \\
\hline
\end{tabular}

Table 2. Aortic diameter in Patients with Ultrasound and Transthoracic EchocardiographyPatients $(\mathrm{n}=122)$

\begin{tabular}{lll}
\hline Aortic diameter & Ultrasound & $\begin{array}{l}\text { Transthoracic } \\
\text { Echocardiography }\end{array}$ \\
\hline $20 \geq$ & $76(62.3)$ & $63(51.6)$ \\
$20-25$ & $34(27.9)$ & $42(34.4)$ \\
$25-30$ & $9(7.4)$ & $14(11.5)$ \\
$\geq 30$ (Abdom. Aortic Aneurysm) & $3(2.5)$ & $3(2.5)$ \\
\hline
\end{tabular}

Table 3. Correlation of some factors with Abdominal Aortic Aneurysm in Patients.

\begin{tabular}{|c|c|c|c|c|}
\hline Factors & Abdominal Aortic & $\begin{array}{l}\text { Aneurysm } \\
\text { Yes N (\%) }\end{array}$ & No N (\%) & P-value \\
\hline Age & $\begin{array}{l}65 \geq \\
65<\end{array}$ & $\begin{array}{l}1(1.7) \\
2(3.2)\end{array}$ & $\begin{array}{l}58(98.3) \\
61(96.8)\end{array}$ & $>0.598$ \\
\hline Sex & $\begin{array}{l}\text { Female } \\
\text { Male }\end{array}$ & $\begin{array}{l}0 \\
3(4.2)\end{array}$ & $\begin{array}{l}51(100) \\
68(95.8)\end{array}$ & $>0.264$ \\
\hline ACS Types & $\begin{array}{l}\text { UA } \\
\text { NSTEMI } \\
\text { Ext. STEMI } \\
\text { Anterior STEMI } \\
\text { Inferior STEMI }\end{array}$ & $\begin{array}{l}2(2.4) \\
1(4 \cdot 5) \\
0 \\
0 \\
0\end{array}$ & $\begin{array}{l}80(97.6) \\
21(95 \cdot 5) \\
8(100) \\
6(100) \\
4(100)\end{array}$ & $>0.931$ \\
\hline Hypertension & $\begin{array}{l}\text { Yes } \\
\text { No }\end{array}$ & $\begin{array}{l}3(7) \\
0\end{array}$ & $\begin{array}{l}40(93) \\
79(100)\end{array}$ & $<0.042$ \\
\hline Smoking & $\begin{array}{l}\text { Yes } \\
\text { No }\end{array}$ & $\begin{array}{l}2(5.7) \\
1(1.1)\end{array}$ & $\begin{array}{l}33(94.3) \\
86(98.9)\end{array}$ & $>0.264$ \\
\hline $\mathrm{LVD}^{*}(\mathrm{~cm})$ & $\begin{array}{l}5 \geq \\
>5\end{array}$ & $\begin{array}{l}0 \\
3(6.4)\end{array}$ & $\begin{array}{l}75(100) \\
44(93.6)\end{array}$ & $<0.027$ \\
\hline
\end{tabular}

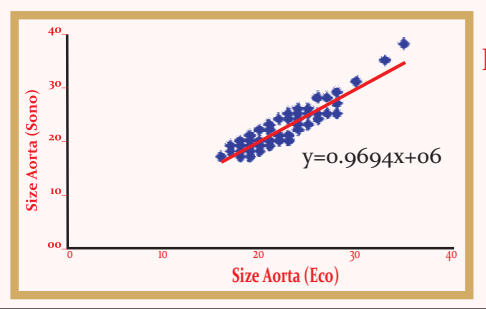

Figure-1: Transthoracic echocardiography aortic size distribution graph based on the Ultrasound in Patients with Acute Coronary Syndrome. 


\section{Discussion:}

Based on the results, the prevalence of abdominal aortic aneurysms in patients with the acute coronary syndrome was $3(2.5 \%)$ based on ultrasonography and echocardiography. Most of them were over 60 years of age, and all of them were male. Also, there was a significant relationship among hypertension, left ventricle size, and abdominal aortic aneurysm. There was no significant relationship among age, sex, type of ACS and smoking in patients with abdominal aortic aneurysm prevalence. The prevalence of abdominal aortic aneurysms in patients with the acute coronary syndrome is low in the present study but is at a high level compared to the general population. According to the literature review, no study has been done to evaluate the prevalence of abdominal aortic aneurysms in patients with acute coronary syndrome based on ultrasonography and echocardiography. The prevalence of abdominal aortic aneurysms in other cardiovascular patients was reported being between 2 and $5 \%$. For example, in the study of Lee et al. (2015), the prevalence of abdominal aortic aneurysms in patients with CAD is $2.4 \%$ based on echocardiography. In a study by Durieux et al. (2014), the incidence of abdominal aortic aneurysms in CAD patients was reported being $4.2 \%$, with an increasing prevalence in men over 65 years old. In a meta-analysis by Elkalioubie et al. (2018), a study of 22 articles including 13388 CAD patients showed that the prevalence of abdominal aortic aneurysms in CAD patients was $8.4 \%$ which was significantly higher than the incidence of abdominal aortic aneurysms in patients without CAD. Smoking, hypertension, and stenosis of the carotid artery are associated with the incidence of abdominal aortic aneurysms in CAD patients. In a meta-analysis by Argyriou et al. (2018), the incidence of abdominal aortic aneurysms was reported to be $0.03 \%$ in the general population based on echocardiography and is higher in males than in females. People with abdominal aortic aneurysm were between 66 and 85 years old. In the study of Dehghan et al. (2010), the prevalence of abdominal aortic aneurysms in CAD patients was $4.3 \%$ based on ultrasonography in Iranian society and age was an important risk factor for an abdominal aortic aneurysm in coronary artery disease.

Regarding the results of the present study and the above studies, it can be argued that the most common cause of an abdominal aortic aneurysm is related to atherosclerotic degeneration of the vascular wall. Risk factors can lead to atherosclerotic degeneration and atherosclerosis, by increasing the pressure of the vascular wall and reducing the vascular wall capacity to resist increased pressure, and thus abdominal aortic aneurysm is created. Therefore, reducing risk factors can reduce abdominal aortic aneurysms (Mohamadifard et al., 2015).

According to the results of the above studies, the study population is one of the important factors in the prevalence difference in various studies. Some studies have included only patients with cardiovascular risk factors, and some others have studied only older people. On the other hand, the prevalence of abdominal aortic aneurysm varies from country to another, depending on the screening method and the size of risk factors, such as heart disease, hypertension, diabetes, smoking, and so on.

Also, based on the most important results of the present study, there was a significant direct correlation between aortic sizes based on sonography and transthoracic echocardiography in patients with acute coronary syndrome. Thus, there is a close diagnostic accuracy between sonography and transthoracic echocardiography in the diagnosis of abdominal aortic aneurysm in patients with acute coronary syndrome. To date, no study has been done to compare the diagnostic accuracy of ultrasonography and transthoracic echocardiography in the diagnosis of abdominal aortic aneurysms in patients with ACS. Thus, it is not possible to compare this study with other studies. Spencer et al. (2004) showed that screening did not reduce the sense of health. A large number of men described their health status similarly or even better than before screening, regardless of whether abdominal aortic aneurysm was found during screening. However, the results of other studies showed that screening abdominal aortic aneurysms could disrupt the quality of life of individuals with abdominal aortic aneurysm and people with a low quality of life before screening (Wanhainen et al., 2004). Annual aortic aneurysms account for $\sim 15,000$ deaths in the United States, out of which 9,0oo were due to abdominal aortic aneurysms (Gillum, 1995). Among those with ruptured abdominal aortic aneurysms, only $10-25 \%$ of the patients could be discharged from the hospital (Bown et al., 2002). The US Preventive Services Task Force has suggested that men aged between 65 and 75 years old who have smoked at any time of their life should do an ultrasound screening once. The loss and share of screening are very close in men aged between 65 and 75 years old who have never smoked so that it is difficult to explain a general conclusion (LeFevre, 2014). Screening of abdominal aortic aneurysm in men over 65 years old is fully compatible with World Health Organization criteria for screening the abdominal aortic aneurysms in the event of a rupture is associated with high mortality. An accepted treatment for it is endovascular repair or endoaneurysmorrhaphy surgery. The disease process is in the form of growth and enlargement of the aneurysm and ultimately its rupture. The latent phase of the disease is very long and asymptomatic so that it remains asymptomatic for many years and, it is shown only with a rupture in many cases. Ultrasonography is a safe and accepted screening method for an abdominal aortic aneurysm. The elective surgery of the abdominal aortic aneurysm in the early stages has a lower mortality rate than in advanced stages compared with 


\section{ORIGINAL ARTICLE}

emergency surgery (Mirsharifi et al., 2011).

Transthoracic echocardiography is usually routine for assessing systolic left ventricular function, disrupted movement of left ventricle wall and prognosis in ACS and CAD patients (Tamborini et al., 2006). Based on the results of this study, echocardiography can be used as a diagnostic screening test in ACS patients, and patients do not require ultrasound, and thus costs are reduced. Since ultrasonography is an additional action in ACS patients, transthoracic echocardiography is done for all patients with ACS. Therefore, two diagnostic modalities are reduced to one modality.

Conclusively, transthoracic echocardiography, a diagnostic screening test can be a suitable substitute for ultrasound in the diagnosis of abdominal aortic aneurysm in patients with the acute coronary syndrome.

\section{References:}

Argyriou, C., Georgiadis, G.S., Kontopodis, N., Pherwani, A.D., Van Herwaarden, J.A., Hazenberg, C. \& Antoniou, G.A. (2018): Screening for abdominal aortic aneurysm during transthoracic echocardiography: a systematic review and meta-analysis. Eur. J. Vasc. Endovasc. Surg., 55(4):475491.

Bown, M.J., Sutton, A.J., Bell, P.R. \& Sayers, R.D. (2002): A metaanalysis of 50 years of ruptured abdominal aortic aneurysm repair. Br. J.Surg., 89(6):714-730.

Buck, D.B., Curran, T., McCallum, J.C., Darling, J., Mamtani, R., van Herwaarden, J.A., Moll, F.L. \& Schermerhorn, M.L. (2016): Management and outcomes of isolated renal artery aneurysms in the endovascular. I. Vasc. Surg., 63(1):7781.

Bush, R.L., Lin, P.H. \& Lumsden, A.B. (2003): Endovascular management of abdominal aortic aneurysms. L. Cardiovasc. Surg., 44(4):527-534.

Durieux, R., Van Damme, H., Labropoulos, N., Yazici, A., Legrand, V., Albert, A., Defraigne, J.O. \& Sakalihasan, N. (2014): High prevalence of abdominal aortic aneurysm in patients with three-vessel coronary artery diseasease. Eur. J. Vasc. Endovasc. Surg., 47(3):273-278.

Elkalioubie, A., Haulon, S., Duhamel, A., Rosa, M., Rauch, A., Staels, B., Susen, S., Van Belle, E. \& Dupont, A. (2015): MetaAnalysis of Abdominal Aortic Aneurysm in Patients With Coronary Artery Disease. Am. J. Cardiol., 116(9):1451-1456.

Gillum, R.F. (1995): Epidemiology of aortic aneurysm in the United States. J. Clin. Epidemiol., 48(11):1289-1298.

Hultgren, R. (2013): Abdominal aortic aneurysms-gender aspects on prevalence, treatment, and concurrent aneurysms. Thorac. Cardiovasc. Surg., 61(1):15-21.
Ambient Science, 2020: Vol. 07h(1); 06-09 DOI:10.21276/ambi.2020.07h.1.oa01

Kontopodis, N., Lioudaki, S., Pantidis, D., Papadopoulos, G., Georgakarakos, E. \& Ioannou, C.V. (2016): Advances in determining abdominal aortic aneurysm size and growth. World J. Radiol., 8(2):148-158.

Kostun, Z.W. \& Malik, R.K. (2016): Screening for abdominal aortic aneurysms. Clin. Imaging, 40(2):321-324.

Lee, S.H., Chang, S.A., Jang, S.Y., Lee, S.C., Song, Y.B., Park, S.W., Choi, S.H., Gwon, H.C., Oh, J.K. \& Kim, D.K. (2015): Screening for abdominal aortic aneurysm during transthoracic echocardiography in patients with significant coronary artery disease. Yonsei Med. J., 56(1):38-44.

LeFevre, M.L. (2014): Screening for abdominal aortic aneurysm: US Preventive Services Task Force recommendation statement. Ann. Intern. Med., 161(4):281-290.

McGregor, J.C., Pollock, J.G. \& Anton, H.C. (1975): The value of ultrasonography in the diagnosis of abdominal aortic aneurysm. Scott. Med. I., 20(3):133-137.

Mirsharifi, R., Zhand, N., Shahriyarian, S., Meysamie, A., Mirsharifi, A. \& Aminian, A. (2011): The prevalence of abdominal aortic aneurysm in Iranian male population over 65 years old. Tehran Uni. Med. J., 69(5):322-326.

Mohamadifard, M., Mamashli, A., Kazemi, T. (2015): Prevalence and risk factors for abdominal aortic aneurysm in people over 65 years old who referred to the ultrasound centers of educational hospitals in Birjand in 2014. L. Birjand Univ. Med. Sci., 22(3):248-255.

Nemati, M., Shakeri, A., Dehghan, R. \& Ghaffari, S. (2009): Prevalence of abdominal aortic aneurysm in patients with coronary artery disease. J. Cardiovasc. Thorac. Res., 1(1):1-4.

Sampson, U.K., Norman, P.E., Fowkes, F.G., Aboyans, V., Song, Y., Harrell, F.E., Jr., Forouzanfar, M.H., Naghavi, M., Denenberg, J.O., McDermott, M.M., Criqui, M.H., Mensah, G.A., Ezzati, M. \& Murray, C. (2014): Estimation of global and regional incidence and prevalence of abdominal aortic aneurysms 1990 to 2010 . Glob. Heart, 9(1):159-170.

Spencer, C.A., Norman, P.E., Jamrozik, K., Tuohy, R. \& LawrenceBrown, M. (2004): Is screening for abdominal aortic aneurysm bad for your health and well-being? ANZ J. Surg., 74(12):1069-1075.

Tamborini, G., Galli, C.A., Maltagliati, A., Andreini, D., Pontone, G., Quaglia, C., Ballerini, G. \& Pepi, M. (2006): Comparison of feasibility and accuracy of transthoracic echocardiography versus computed tomography in patients with known ascending aortic aneurysm. Am. J. Cardiol., 98(7):966969.

Wanhainen, A., Rosen, C., Rutegard, J., Bergqvist, D. \& Bjorck, M. (2004): Low quality of life prior to screening for abdominal aortic aneurysm: a possible risk factor for negative mental effects. Ann. Vasc. Surg., 18(3):287-293. 